\title{
Exogenous carbon monoxide suppresses Escherichia coli vitality and improves survival in an Escherichia coli-induced murine sepsis model
}

\author{
Wei-chang SHEN", Xu WANG\#, Wei-ting QIN, Xue-feng QIU, Bing-wei SUN* \\ Department of Burns and Plastic Surgery, Affiliated Hospital, Jiangsu University, Zhenjiang 212001, China
}

\begin{abstract}
Aim: Endogenous carbon monoxide $(\mathrm{CO})$ has been shown to modulate inflammation and inhibit cytokine production both in vivo and in vitro. The aim of this study was to examine whether exogenous carbon monoxide could suppress the vitality of Escherichia coli (E coli) and improve the survival rate in an $E$ coli-induced murine sepsis model.

Methods: ICR mice were infected with $E$ coli, and immediately injected intravenously with carbon monoxide releasing molecule-2 (CORM-2, $8 \mathrm{mg} / \mathrm{kg})$ or inactive CORM-2 $(8 \mathrm{mg} / \mathrm{kg})$. The survival rate was monitored 6 times daily for up to $36 \mathrm{~h}$. The blood samples, liver and lung tissues were collected at $6 \mathrm{~h}$ after the infection. Bacteria in peritoneal lavage fluid, blood and tissues were enumerated following culture. Tissue iNOS mRNA expression was detected using RT-PCR. NF-kB expression was detected with Western blotting. Results: Addition of CORM-2 (200 and $400 \mu \mathrm{mol} / \mathrm{L}$ ) into culture medium concentration-dependently suppressed the growth of $E$ coli and decreased the colony numbers, but inactive CORM-2 had no effect. Treatment of the infected mice with CORM-2 significantly increased the survival rate to 55\%, while all the infected mice treated with inactive CORM-2 died within $36 \mathrm{~h}$. E coli infection caused severe pathological changes in liver and lungs, and significantly increased serum transaminases, lipopolysaccharide, TNF- $\alpha$ and IL-1 $\beta$ levels, as well as myeloperoxidase activity, TNF- $\alpha$ and IL-1 $\beta$ levels in the major organs. Meanwhile, $E$ coli infection significantly increased the number of colonies and the expression of iNOS mRNA and NF-kB in the major organs. All these abnormalities were significantly attenuated by CORM-2 treatment, while inactive CORM-2 was ineffective.

Conclusion: In addition directly suppressing $E$ coli, CORM-2 protects the liver and lungs against $E$ coli-induced sepsis in mice, thus improving their survival.
\end{abstract}

Keywords: sepsis; Escherichia coli; carbon monoxide; CO-releasing molecule-2; liver; lung; cytokine; lipopolysaccharide; iNOS; NF-kB; inflammation

Acta Pharmacologica Sinica (2014) 35: 1566-1576; doi: 10.1038/aps.2014.99; published online Nov 172014

\section{Introduction}

Sepsis is a clinical syndrome that complicates severe infection ${ }^{[1]}$. A subset of patients develops multiple organ dysfunction syndrome (MODS) rapidly leading to death, while another subset goes into remission but is more prone to develop secondary life-threatening microbial infections ${ }^{[2,3]}$. Sepsis and severe sepsis are leading causes of death in many intensive care units (ICU) all around the world ${ }^{[4]}$. Recently in the United States, the incidence of severe sepsis has been estimated to be $3 \%$ of the population, with a death rate of $25 \%$ among these cases during hospitalization ${ }^{[5]}$. Clinically, nearly $95 \%$ of sepsis cases are caused by bacterial infection, and $62.2 \%$ of these can be

\footnotetext{
\# These authors contributed equally to this work.

* To whom correspondence should be addressed.

E-mail sunbinwe@hotmail.com

Received 2014-06-10 Accepted 2014-08-20
}

attributed to Gram-negative bacteria, with Escherichia coli ( $E$ coli) responsible for nearly $16 \%$ of these infections ${ }^{[6]}$. E coli is an opportunistic pathogen, which migrates by bacterial translocation from the gastrointestinal tract to extra-intestinal sites, where virulence factors are important in establishing infections. In recent years, more frequent use of broad-spectrum antibiotics has increased bacterial resistance ${ }^{[7,8]}$; therefore, novel and effective antibacterial agents for use in the clinical are urgently required.

Carbon monoxide (CO) is a stable gas that has long been known as a toxic compound because of its ability to bind hemoglobin. Endogenous carbon monoxide, which is a byproduct of inducible heme oxygenase-1 (HO-1), can modulate inflammation and inhibit lipopolysaccharide (LPS)-induced production of cytokines both in vivo and in vitro ${ }^{[9-11]}$. Recently, transitional metal carbonyls have been identified as potential CO-releasing molecules (CORMs) with the potential to deliver 
CO to tissues and organs as a therapeutic agent ${ }^{[12,13]}$. We have reported that CORM-2 attenuated leukocyte sequestration in some vital organs and the small intestine in burned and cecal ligation and puncture (CLP)-challenged mouse models of sepsis through interfering with nuclear factor-kB (NF-kB) activation and intercellular adhesion molecule-1 (ICAM-1) protein expression and suppressing the endothelial cell proadhesive phenotype ${ }^{[14-17]}$. In addition, our earlier studies also showed that CORM-2 inhibits the activity and virulence of $E$ coli in vitro. On the basis of these observations, the aim of the present study was to determine the capacity of exogenous $\mathrm{CO}$, carbon monoxide-releasing molecule II (CORM-2), to suppress E coli ATCC25922 vitality, and to decrease the inflammatory responses in the liver and lung, increase the survival rate in an E coli ATCC25922-induced murine model of sepsis.

\section{Materials and methods}

\section{Ethics statement}

The experimental protocol was approved by the Council on Animal Care at Jiangsu University on the Protection and Welfare of Animals and was conducted according to the National Institutes of Health guidelines for the care and use of experimental animals. Anesthesia consisting of spontaneous inhalation of isoflurane- $\mathrm{N}_{2} \mathrm{O}$ (Abbott Laboratories, Missisauga, Ontario, Canada) in a $60 \%$ oxygen $/ 40 \%$ nitrogen mixture was administered when necessary. Mice were euthanized by excessive anesthesia administration for each experiment.

\section{Reagents}

Tricarbonyldichlororuthenium (II) dimer (carbon monoxide releasing molecule-2, CORM-2) was purchased from Sigma (St Louis, Mo, USA) and solubilized in dimethyl sulfoxide (DMSO) to obtain a $40 \mathrm{mmol} / \mathrm{L}$ stock solution. Chemical structure of CORM-2 was shown in our published report ${ }^{[18]}$. Inactive form of CORM-2 (iCORM-2, negative controls) was prepared as described previously ${ }^{[18]}$. E coli ATCC25922 was kindly offered by Department of Microbiological Testing of the Affiliated Hospital of Jiangsu University. Other reagents and instruments included tumor necrosis factor-alpha (TNFa) and interleukin-1 beta (IL-1 $\beta$ ) ELISA kits (Qiaoyi, Shanghai, China), LAL reagents (Houshiji, Fujian, China), a high-speed and low-temperature desktop centrifuge (Beckman, Inc, USA), and a VMR-type small animal anesthesia machine (Matrx, Inc, USA), a CFX96TM Real-Time System (Bio-Rad, Inc, USA). All the other chemicals were of reagent grade and obtained from Sigma unless otherwise stated.

\section{Growth curve measuring and colony counting}

Measurement of growth curve was used to dynamically study the growth of bacteria. The bacterial growth data in our preliminary experiments indicated that the consistent major effect of CORM-2 on growth dynamics was an extended lag phase in which entry into exponential growth was delayed that increased with increasing CORM-2 concentrations. Thus, we focused on how CORM-2 altered the duration of the lag phase. The bacteria were divided into five groups $(n=6)$, including
E coli, E coli+200 $\mu \mathrm{mol} / \mathrm{L}$ CORM-2, E coli+400 $\mu \mathrm{mol} / \mathrm{L}$ CORM2, E coli+200 $\mu \mathrm{mol} / \mathrm{L}$ iCORM-2, and $E$ coli $+400 \mu \mathrm{mol} / \mathrm{L}$ iCORM-2. E coli were inoculated and cultured on the LuriaBertani (LB) medium until they reached peak viability and activity. Single colonies were then inoculated into $2 \mathrm{~mL}$ of LB medium, cultured overnight $\left(37^{\circ} \mathrm{C}\right.$ at $\left.250 \mathrm{r} / \mathrm{min}\right)$, diluted at the rate of 1:100, and inoculated in $20 \mathrm{~mL}$ of LB medium in the following morning. Corresponding concentrations of CORM-2 or iCORM-2 (200 or $400 \mu \mathrm{mol} / \mathrm{L}$ ) were added to each group (except $E$ coli group) to intervene, and the values at $O D_{600}$ were measured at specific time points. In addition, $100 \mu \mathrm{L}$ of the bacterial culture was appropriately diluted and evenly spread on Mueller-Hinton agar plates. The bacteria were cultured in a $\mathrm{CO}_{2}$ incubator at $37^{\circ} \mathrm{C}$ for $48 \mathrm{~h}$ and the resulting colonies were then counted.

\section{Infection model and survival rate}

Pathogen-free male ICR mice (kindly offered by Laboratory Animal Center, Yangzhou University) aged 6-8 weeks and weighing $20 \pm 2 \mathrm{~g}$ were fed in laboratory for one week. Mice were housed in standard wire-topped cages and in temperature-controlled units. Food and water were supplied ad libitum. A total of 80 ICR mice were divided into four groups $(n=20)$ as follows: control, E coli, E coli+CORM-2, E coli+iCORM-2. The control group received $0.5 \mathrm{~mL}$ saline by intraperitoneal injection, while the other groups received intraperitoneal injections of $0.5 \mathrm{~mL} \mathrm{E}$ coli $\left(3 \times 10^{8} \mathrm{cfu} / \mathrm{mL}\right)$. Immediately afterwards, the E coli+CORM-2 and E coli+iCORM-2 groups received CORM-2 (8 mg/ kg, iv) or iCORM-2 $(8 \mathrm{mg} / \mathrm{kg}$, iv), respectively. The concentration of CORM-2 used in the present study was based on a previous report of the use of this compound in mice and our own preliminary experiments that measured the dynamic and peak carboxyhemoglobin $(\mathrm{COHb})$ levels ${ }^{[19]}$. Mouse survival was monitored six times daily for up to $36 \mathrm{~h}$.

\section{Histologic studies}

A total of 32 ICR mice were divided into four groups $(n=8)$ and were infected with E coli with CORM-2 or iCORM-2 intervention as described above. Six hours after the injection, the mice were euthanized by excessive anesthesia administration. The liver and lung specimens harvested from different groups of mice were fixed by immersion in $4 \%$ formaldehyde solution. The tissues were embedded in paraffin wax, serially sectioned, and stained with hematoxylin-eosin. Liver and lung morphologic characteristics were evaluated by light microscopy.

\section{LPS concentration assessment}

The concentration of LPS in the serum of mice was determined using the Limulus amebocyte lysate (LAL) test (Houshiji, Fujian, China). In accordance with the protocol provided by the manufacturer, $100 \mu \mathrm{L}$ of each sample was added into 100 $\mu \mathrm{L}$ of the quantitative LAL reagents dissolved in LPS-free water and then incubated in a motionless water bath at $37^{\circ} \mathrm{C}$ for $2 \mathrm{~h}$. The formation of gel from the LAL products induced by the existence of non-neutralized LPS was measured utiliz- 
ing the kinetic turbidimetric assay. The results were analyzed using GEN5 data analysis software.

\section{Serum transaminase levels}

Blood samples were obtained by cardiac puncture of the left ventricle. The samples were stored in serum tubes and immediately centrifuged at $3000 \mathrm{~g} / \mathrm{min}$ for $5 \mathrm{~min}$. Evaluation of hepatocellular injury was performed by determining the enzymatic activity of ALT and AST in serum samples using a commercially available kit (Boehringer Manheim, Munich, Germany) according to the manufacturer's instructions.

\section{TNF- $\alpha$ and IL-1 $\beta$ levels in serum and tissue homogenates}

TNF- $\alpha$ and IL-1 $\beta$ levels in serum, and tissue homogenates (liver and lung) were measured by enzyme-linked immunosorbent assay (ELISA) kits following the manufacturer's instructions.

\section{Myeloperoxidase (MPO) activity}

MPO activity was measured in liver and lung tissues using a procedure similar to that documented by Hillegas et al ${ }^{[20]}$. Tissue samples were homogenized in $50 \mathrm{mmol} / \mathrm{L}$ potassium phosphate buffer (PB) ( $\mathrm{pH} 6.0)$, and centrifuged at $10000 \times g$ (10 min); pellets were suspended in $50 \mathrm{mmol} / \mathrm{L} \mathrm{PB}$ containing $0.5 \%$ hexadecyltrimethylammonium bromide. After sonication, the samples were centrifuged at $10000 \times \mathrm{g}$ (10 $\mathrm{min})$. Aliquots $(0.3 \mathrm{~mL})$ were added to $2.3 \mathrm{~mL}$ of reaction mixture containing $50 \mathrm{mmol} / \mathrm{L} \mathrm{PB}, o$-dianisidine, and $20 \mathrm{mmol} / \mathrm{L}$ $\mathrm{H}_{2} \mathrm{O}_{2}$ solution. One unit of enzyme activity was defined as the amount of MPO present that caused a change in absorbance measured at $460 \mathrm{~nm}$ for $3 \mathrm{~min}$. MPO activity was expressed as unit/g tissue.

\section{Colony counting}

Mice were infected by $E$ coli followed by immediate intravenous administration of CORM-2 or iCORM-2 as described above. Six hours after the infection, the mice were euthanized by excessive anesthesia administration. Samples $(100 \mu \mathrm{L})$ of the blood, peritoneal lavage fluid, liver and lung homogenates of the mice were appropriately diluted and evenly spread on Mueller-Hinton agar plates, cultured in a $\mathrm{CO}_{2}$ incubator at $37^{\circ} \mathrm{C}$ for $24 \mathrm{~h}$. Finally the resulting colonies were counted.

\section{Semiquantitation of iNOS mRNA by reverse transcription- polymerase chain reaction (RT-PCR)}

The liver and lung tissue samples were sheared and treated with Trizol, chloroform, isopropanol, ethanol, and RNase free water in order to extract total RNA. Reversed transcription of RNA to yield cDNA was carried out using Revert Aid First Strand cDNA Synthesis Kit (Thermo, Boston, USA). Quantitative real-time PCR (Maxima SYBR Green/ROX qPCR Kit, Thermo) amplification was performed under the conditions of $95^{\circ} \mathrm{C}$ for $10 \mathrm{~min}$ and $95^{\circ} \mathrm{C}$ for $10 \mathrm{~s}$, followed by $1 \mathrm{~min}$ at $60^{\circ} \mathrm{C}$, with data collection during the last $30 \mathrm{~s}$. Primer sequences for the genes of interest and the reference gene (GAPDH) were as follows: for iNOS, forward primer 5'-CTTCCGAAGTTTCTG-
GCAGCAGCG-3' and reverse primer 5'-GAGCCTCGTGGCTTTGGGCTCCTC-3'; for GAPDH, forward primer 5'-CACCCCATTTGATGTTAGTGC-3' and reverse primer 5'-CCATTTGCAGTGGCAAAG-3'. The values were standardized by comparing with values from relative expression of GAPDH.

\section{Western blot}

Nucleic proteins were extracted with the nuclear protein extraction buffer kit (Vazyme Biotech, Jiangsu, China). Protein concentration was assayed using the bicinchoninic acid (BCA) protein assay kit (Beyotime, Jiangsu, China) and equal amounts of protein were loaded per well on $7.5 \%-15 \%$ polyacrylamide gels. An anti-mouse NF-кB-specific polyclonal antibody (1:200, Santa Cruz Biotechnology, Santa Cruz, CA, USA) was used as the primary antibody and an HRP-conjugated goat anti-rabbit IgG antibody (Santa Cruz Biotechnology, Santa Cruz, CA, USA) was used as secondary antibody at the appropriate concentration (1:2500). Immunoreactive bands were visualized using an ECL detection system and band intensity was determined using Fluorchem FC3 software.

\section{Statistical analysis}

Statistical analyses were performed by using SPSS 17 software (SPSS, Chicago, IL, USA). Data were expressed as mean \pm SD. One-way analysis of variance (ANOVA) and the independentsamples $t$-test was used for the comparisons. A value of $P<0.05$ was considered to be statistically significant.

\section{Results}

Effects of CORM-2 on E coli growth curve and colony counting $E$ coli were divided into five groups $(n=6)$. Each group (except E coli group) was added with CORM-2 or iCORM-2 to coculture at corresponding concentrations (200 or $400 \mu \mathrm{mol} / \mathrm{L}$ ), and strain growth was monitored at hourly intervals until stationary phase was reached. Growth was monitored by both determining the optical density at $600 \mathrm{~nm}\left(O D_{600}\right)$ and counting the clonal formation unit (CFU). Bacterial growth was markedly suppressed in the presence of CORM-2. This was confirmed by observing growth curve of $E$ coli under intervention of CORM-2 or iCORM-2. When compared with E coli and $E$ coli+iCORM-2 group, the growth of $E$ coli in $E$ coli +200 $\mu \mathrm{mol} / \mathrm{L}$ CORM-2 group was suppressed, and the platform phase entering time was significantly delayed, with a decrease in colony numbers ( $F$ value in Figure $1 \mathrm{~A}$ and $1 \mathrm{~B}$ were 1034.33 and 248.32 respectively, $P<0.01)$. Group $E$ coli $+400 \mu \mathrm{mol} / \mathrm{L}$ CORM-2 showed an even more obvious suppression of bacterial growth and decrease in colony numbers ( $F$ value in Figure $1 C$ and D were 7087.46 and 11342.67 respectively, $P<0.01)$.

\section{Effect on CORM-2 on survival}

All mice that received intraperitoneal injections of $E$ coli alone died within $36 \mathrm{~h}$, while CORM-2 administration significantly increased the survival rate which reached 55\% $(P<0.05)$. All mice that received iCORM-2 immediately after intraperitoneal injections of $E$ coli also died within $36 \mathrm{~h}$ (Figure 2). 

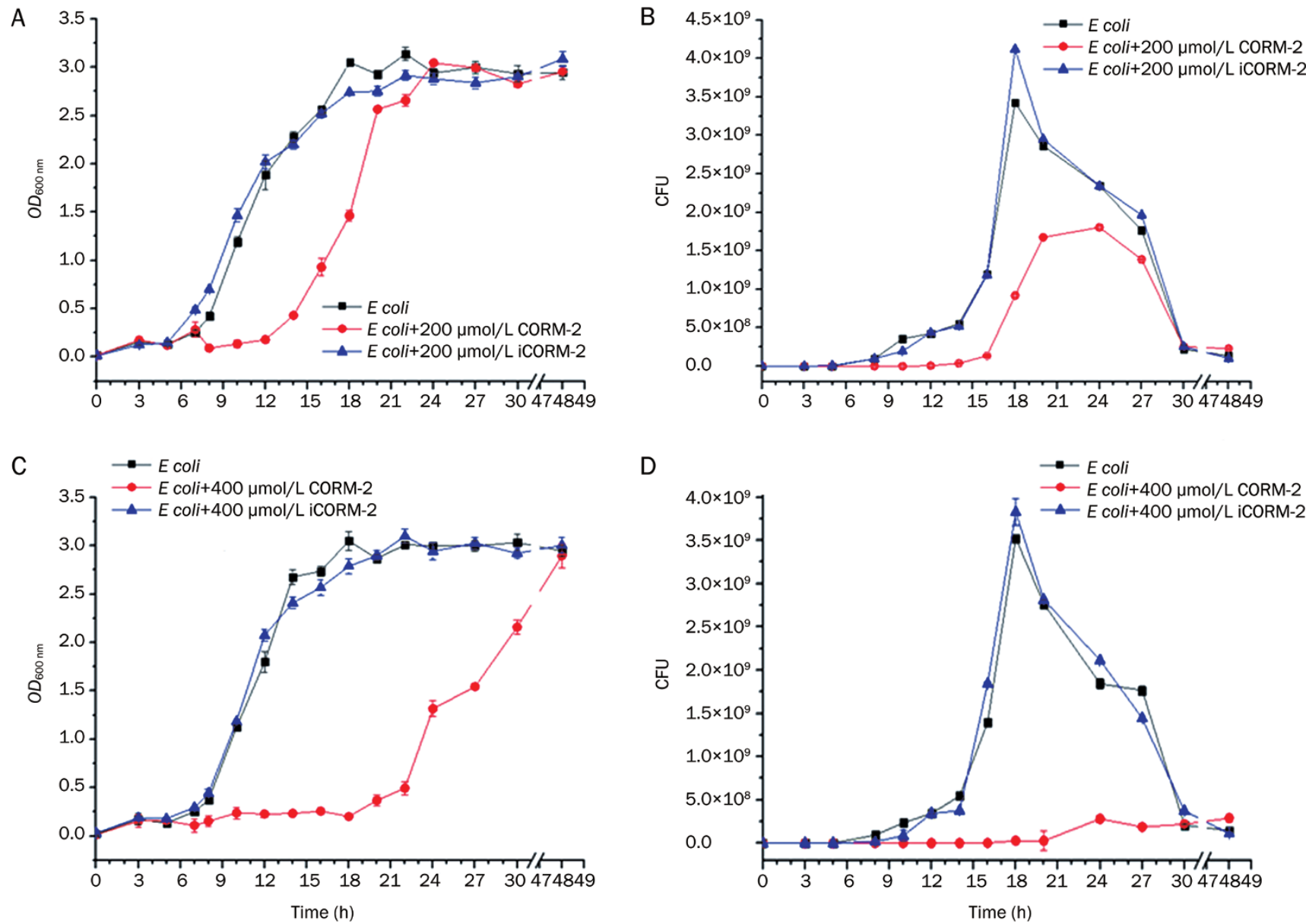

Figure 1. Effects of CORM-2 on the growth curve and colony counting of $E$ coli. The bacteria culture was appropriately diluted and evenly spread on Mueller-Hinton agar plates. Bacteria were cultured in $\mathrm{CO}_{2}$ incubator at $37^{\circ} \mathrm{C}$. CORM-2 was added to the bacteria culture at the concentration of 200 or $400 \mu \mathrm{mol} / \mathrm{L}$ to observe its effects on the $E$ coli growth curve and resulting colonies. When compared to group $E$ coli, the bacterial growth was significantly suppressed (A, C) and colony numbers markedly decreased (B, D) in group E coli+200 $\mu \mathrm{mol} / \mathrm{L}$ CORM-2 and in group E coli+400 $\mu \mathrm{mol} / \mathrm{L}$ CORM-2 ( $F=1034.33,248.32$, and 7087.46, 11342.67, respectively, $P<0.01) . n=8$.

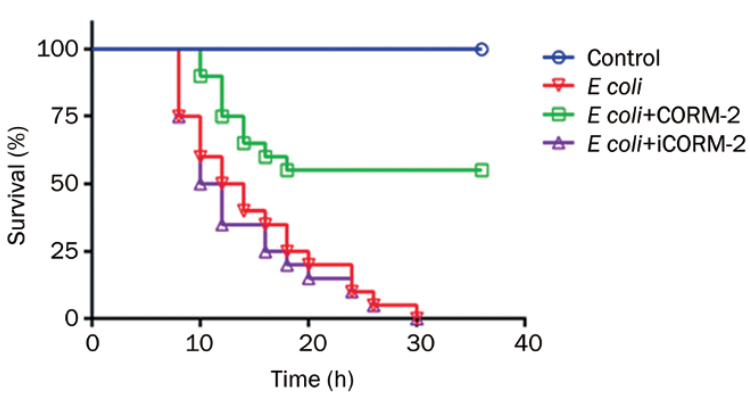

Figure 2. Effects of CORM-2 on survival in an E coli-induced murine model of sepsis. Mice were divided into four groups as follows: control (saline), E coli, E coli+CORM-2, E coli+iCORM-2. Mice were infected by $E$ coli followed by immediate intravenous administration of CORM-2 or iCORM-2. All $E$ coli infected mice and $E$ coli infected mice with iCORM-2 treatment died within $36 \mathrm{~h}$, while CORM-2 administration significantly increased the survival rate which reached $55 \%$. $n=8$.

\section{Pathological changes}

The organ sections of the animals in each group were evaluated microscopically using $\mathrm{H}+\mathrm{E}$ staining. Histological analysis revealed normal structure in the liver and lung sections from the control group (Figure 3A, 3E). In the $E$ coli infected mice, marked vacuolization and infiltration of the liver by inflammatory cells was observed (Figure 3B). Increased numbers of red blood cells and inflammatory cells were also observed in the lung alveolae (Figure 3F). These morphological changes in both lung and liver suggested the presence of systemic inflammation and multiple organ damage after $E$ coli challenge, and these pathological changes also existed in tissues of E coli $+\mathrm{iCORM}-2$ group (Figure 3D, 3H). After in vivo administration of CORM-2, granulocyte infiltrations were significantly decreased in both liver and lung tissue (Figure 3C, 3G), providing evidence that CORM-2 may be involved in the suppressive effects against $E$ coli vitality and toxicity. The figure 

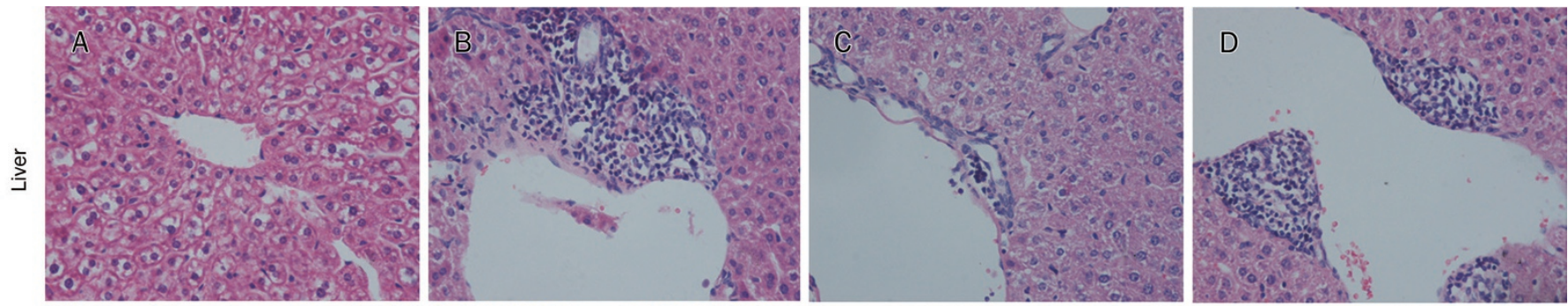

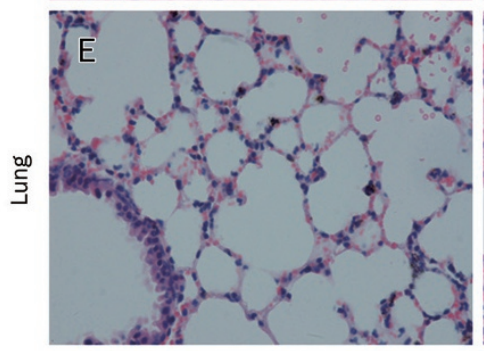

Control

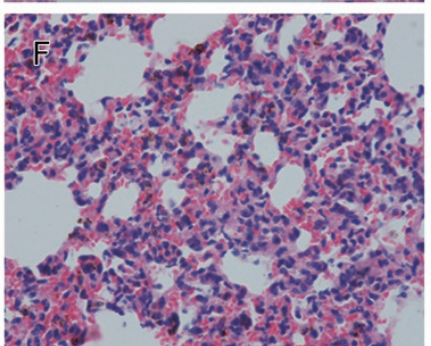

E coli

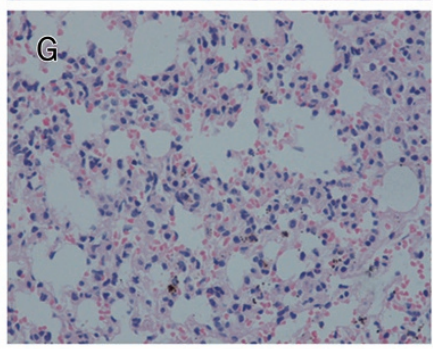

E coli+CORM-2

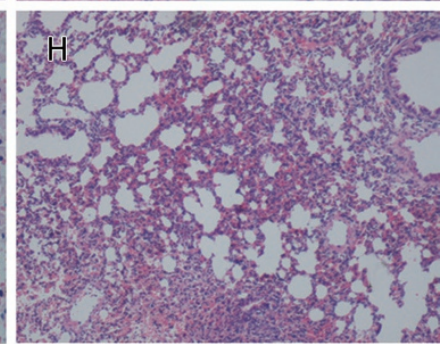

E coli+iCORM-2

Figure 3. Effects of CORM-2 on organs injury after $E$ coli infection. Mice were infected by $E$ coli followed by immediate intravenous administration of CORM-2 or iCORM-2. After $6 \mathrm{~h}$ of infection, the liver and lung specimens harvested from different groups of mice were immersed in $4 \%$ formaldehyde solution. The tissues were embedded in paraffin wax, serially sectioned, and stained with hematoxylin-eosin. Tissue morphologic characteristics were evaluated under light microscope. (A, E) Sections from control mice had normal architecture of the liver and lung; (B, F) Sections from bacteria infected mice showed granulocyte infiltrations were significantly increased in liver and lung at $6 \mathrm{~h}$ of $E$ coli infection; (C, G) Section from bacteria infected mice followed by immediate intravenous administration of CORM-2 showed that histological damage in lung and liver tissue and infiltration of the inflammatory cells to the tissue were significantly attenuated. The figure is representative of at least three experiments performed on different days. (D, F) Pathologic changes in the tissues of $E$ coli+iCORM-2 group were very similar to that of $E$ coli group.

is representative of at least three experiments performed on different days.

\section{Effect on CORM-2 on organ function and MPO activity in liver and lung tissues}

Hepatocyte injury was evaluated by determining the serum concentrations of ALT and AST. At $6 \mathrm{~h}$ after receiving intraperitoneal injections of $E$ coli, serum ALT and AST were found to be significantly elevated in E coli and E coli+iCORM-2 infected mice as compared to the mice of control group (ALT, $131.50 \pm 12.93$ and $125.00 \pm 11.33$ vs $44.18 \pm 2.76$; AST, $244.63 \pm 22.61$ and $237.75 \pm 21.78$ vs $55.25 \pm 5.01 \mathrm{U} / \mathrm{L}, P<0.05)$. In contrast, the corresponding values in the $E$ coli+CORM-2 group were significantly lower than those in the $E$ coli and $E$ colitiCORM-2 groups (ALT, $87.13 \pm 6.42$ vs $131.50 \pm 12.93$ and $125.00 \pm 11.33$ $\mathrm{U} / \mathrm{L}$; AST, $172.68 \pm 13.40$ vs $244.63 \pm 22.61$ and $237.75 \pm 21.78 \mathrm{U} / \mathrm{L}$, $P<0.05$, Figure $4 \mathrm{~A}$ ).

The activity of MPO, which is an enzyme in the azurophilic granules of neutrophils, was assessed to determine whether the $E$ coli-induced polymorphonuclear leukocyte (PMN) accumulation in the liver and lung was effectively prevented by treatment with CORM-2. Homogenates of the organ samples
A

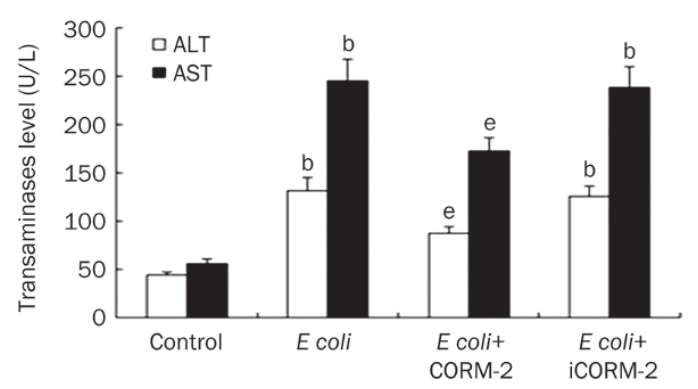

B

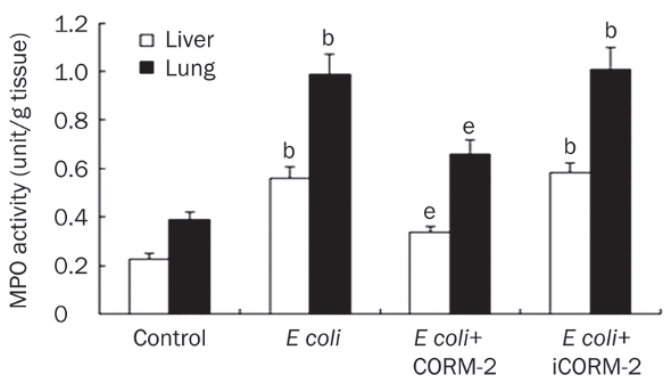

Figure 4. Effect on CORM-2 on organ function and MPO activity in liver and lung tissues. Mice were infected by $E$ coli followed by immediate intravenous administration of CORM-2 or iCORM-2. Serum ALT and AST levels and MPO activity in liver and lung tissues were measured 6 h after $E$ coli infection. Serum ALT, AST, and MPO activities in liver and lung tissues were found to be significantly elevated in $E$ coli infected mice as compared to the mice of control group (A, B). In contrast, the corresponding values in the $E$ coli+CORM-2 group were significantly lower than those in the $E$ coli and $E$ coli+iCORM-2 groups (A, B). Data represent the mean \pm SD. ${ }^{b} P<0.05$ compared with the control group. ${ }^{e} P<0.05$ compared with the $E$ coli group. 
A

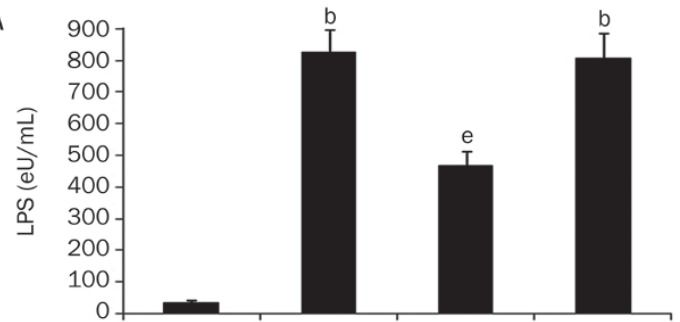

C

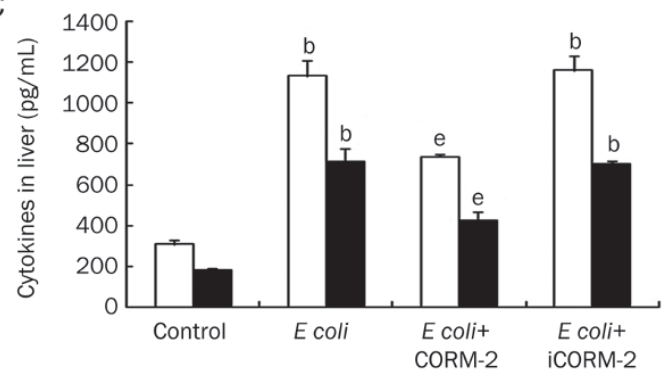

B

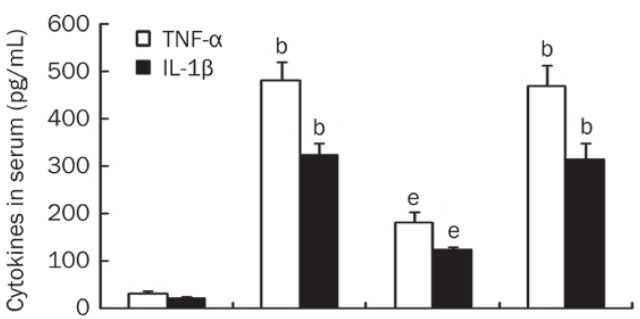

D

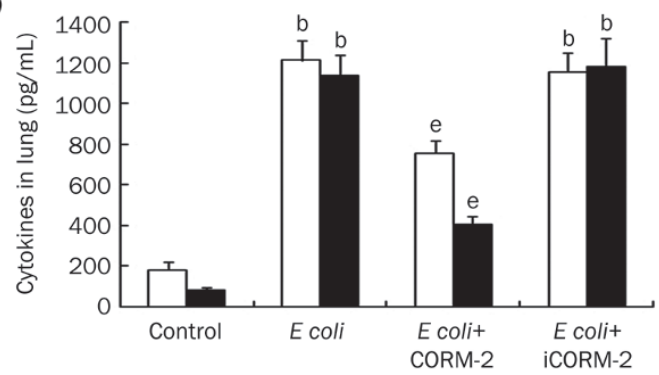

Figure 5. Effect of CORM-2 on LPS concentrations and cytokines in serum and tissue homogenates. Mice were infected by $E$ coli followed by immediate intravenous administration of CORM-2 or iCORM-2. At $6 \mathrm{~h}$ after $E$ coli infection, serum concentrations of LPS increased significantly after bacterial infection compared with control group, while the concentration of LPS in the E coli+CORM-2 group was significantly lower than that detected in the $E$ coli and $E$ coli+iCORM-2 groups (A). The TNF- $\alpha$ and IL-1 $\beta$ levels in the serum as well as in the liver and lung homogenates of $E$ coli and $E$ coli+iCORM-2 mice were significantly increased compared to the levels detected in the control group. In the $E$ coli+CORM-2 group, the elevation in serum and tissue homogenate levels of TNF- $\alpha$ and IL-1 $\beta$ was significantly reduced (B-D). $n=8$. Data represent the mean \pm SD. ${ }^{b} P<0.05$ compared with the control group. ${ }^{\mathrm{e}} \mathrm{P}<0.05$ compared with the $E$ coli group.

were examined for MPO activity at $6 \mathrm{~h}$ after bacterial infection. MPO activity in liver and lung homogenates obtained from $E$ coli and E coli+iCORM-2 infected mice was markedly increased compared with control mice (liver, $0.56 \pm 0.05$ and $0.59 \pm 0.04$ vs $0.28 \pm 0.02$ unit/g; lung, $0.99 \pm 0.08$ and $1.01 \pm 0.09$ vs $0.39 \pm 0.03$ unit $/ g, P<0.05)$, while this increase was significantly reduced by CORM-2 treatment (liver, $0.34 \pm 0.02$ vs $0.56 \pm 0.05$ and $0.59 \pm 0.04$ unit/g; lung, $0.66 \pm 0.06$ vs $0.99 \pm 0.08$ and $1.01 \pm 0.09$ unit/g, $P<0.05$, Figure $4 \mathrm{~B}$ ).

Effect of CORM-2 on LPS concentration and cytokines levels in serum, liver and lung

Mice were infected by $E$ coli followed by immediate intravenous administration of CORM-2 or iCORM-2. At $6 \mathrm{~h}$ after receiving intraperitoneal injection of $E$ coli, serum concentrations of LPS increased significantly after bacterial infection compared with control group $(826.51 \pm 71.98$ vs $36.35 \pm 8.42$ $\mathrm{eU} / \mathrm{mL}, P<0.05)$, while the concentration of LPS in the $E$ coli+CORM-2 group was significantly lower than that detected in the $E$ coli and $E$ coli $+\mathrm{iCORM}-2$ groups $(468.97 \pm 43.29$ vs $826.51 \pm 71.98$ and $805.39 \pm 81.22 \mathrm{eU} / \mathrm{mL}, P<0.05$, Figure $5 \mathrm{~A}$ ). In parallel, the TNF- $\alpha$ and IL-1 $\beta$ levels in the serum as well as in the liver and lung homogenates of $E$ coli and E coli+iCORM-2 mice were significantly increased compared to the levels detected in the control group. In the E coli+CORM-2 group, the elevation in serum and tissue homogenate levels of TNF- $\alpha$ and IL-1 $\beta$ was significantly reduced compared with $E$ coli and E coli+iCORM-2 group mice $(P<0.05$, Figure 5B-5D).
Detection of colony distribution in major mouse organs after E coli infection

Mice were infected by $E$ coli followed by immediate intravenous administration of CORM-2 or iCORM-2. Six hours after the injection, the mice were killed by excessive anesthesia. The number of colonies in blood, peritoneal lavage fluid (PLF), liver and lung homogenates of $E$ coli infected mice were significantly increased compared with that in the control group $(P<0.001)$, while the number of colonies in the major organs of $E$ coli+CORM-2 group were significantly decreased compared with that in the $E$ coli group [blood, $(1.21 \pm 0.50) \times 10^{6}$ us $(5.25 \pm 4.90) \times 10^{7}$ and $(4.10 \pm 3.30) \times 10^{7} \mathrm{cfu} / \mathrm{mL}$; PLF, $(2.16 \pm 1.38) \times 10^{7}$ vs $(2.71 \pm 2.00) \times 10^{10}$ and $(2.68 \pm 2.10) \times 10^{10}$ $\mathrm{cfu} / \mathrm{mL}$; liver, $(1.47 \pm 1.11) \times 10^{6}$ vs $(4.92 \pm 3.20) \times 10^{7}$ and $(5.40 \pm 4.70) \times 10^{7} \mathrm{cfu} / \mathrm{mL}$; lung, $(3.90 \pm 3.30) \times 10^{6}$ vs $(6.94 \pm 3.92) \times 10^{7}$ and $(6.23 \pm 4.34) \times 10^{7} \mathrm{cfu} / \mathrm{mL}, P<0.01$. Figure 6].

Effect of CORM-2 on iNOS mRNA expression in the liver and lung of $E$ coli infected mice

Mice were infected by $E$ coli followed by immediate intravenous administration of CORM-2 or iCORM-2. Six hours after the injection, the expressions of iNOS mRNA in liver and lung of E coli and E coli+iCORM-2 group mice significantly increased compared to that of the control group (liver, $32.99 \pm 2.96$ and $33.65 \pm 3.29$ vs $1 \pm 0$; lung, $19.01 \pm 2.71$ and $18.15 \pm 2.60$ vs $1 \pm 0, P<0.05)$. This increase was significantly reduced in the $E$ coli+CORM-2 group (liver, $13.54 \pm 3.17$ vs $32.99 \pm 2.96$; lung, $6.74 \pm 0.79$ vs $19.01 \pm 2.71, P<0.05$, Figure 7 ). 

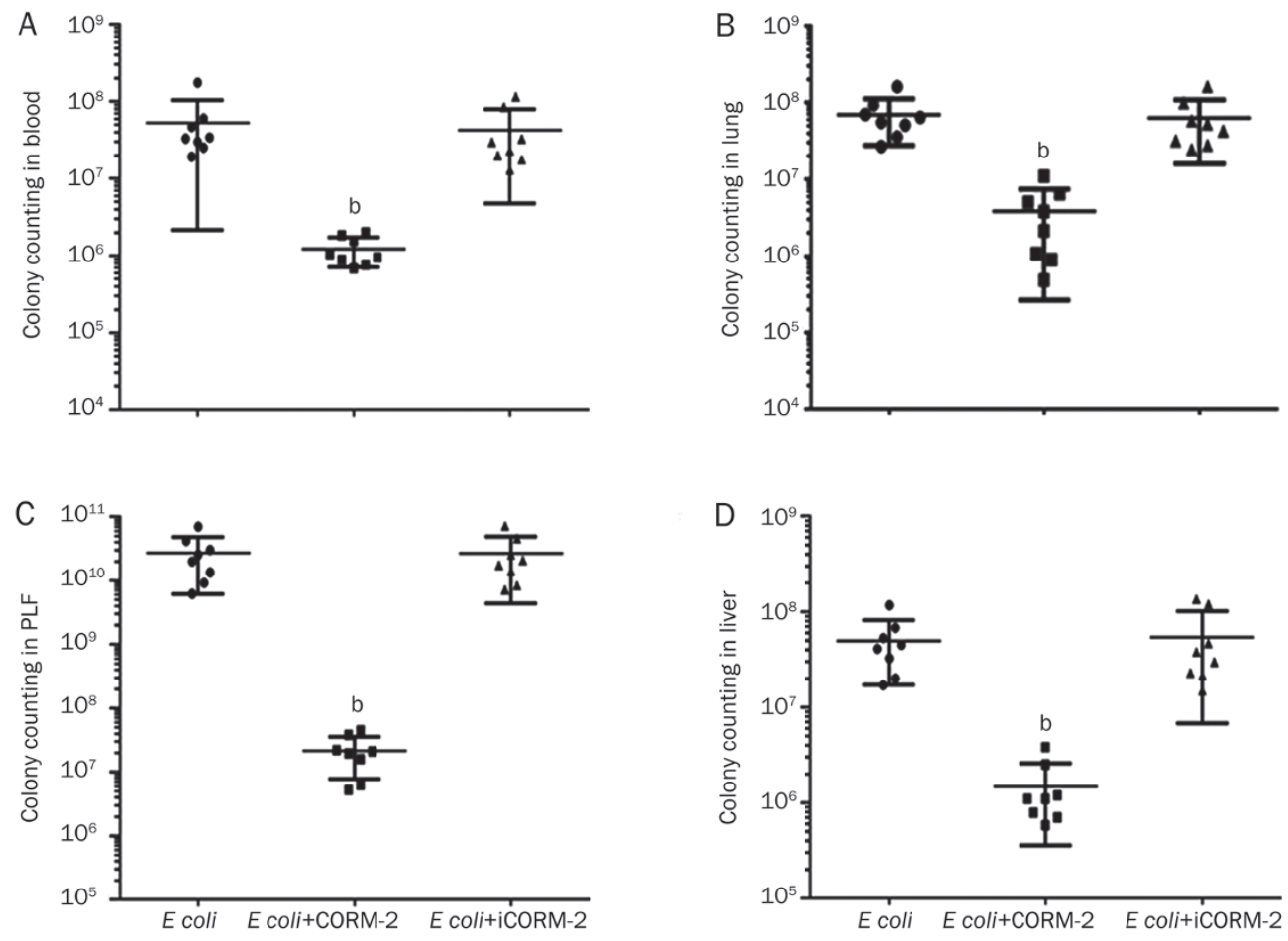

Figure 6. Effect of CORM-2 on colony distribution in major mouse organs after $E$ coli infection. Mice were infected by $E$ coli and treated with CORM-2 or iCORM-2. Six hours after the injection, the mice were killed by excessive anesthesia. Mice blood, peritoneal lavage fluid (PLF) and liver, lung homogenates were prepared. Appropriately diluted homogenates were spread onto LB plates, and cultured in $\mathrm{CO}_{2}$ incubator at $37^{\circ} \mathrm{C}$ for $24 \mathrm{~h}$, followed with colony counting. The colony counting in blood (A), lung (B), PLF (C), and liver (D) homogenates of $E$ coli infected mice were significantly increased, while the colony counting in major organs in $E$ coli+CORM-2 groups were significantly decreased compared with that in $E$ coli group. $n=8$. Data represent the mean \pm SD. ${ }^{\mathrm{b}} P<0.01$ compared with the $E$ coli group.

Effect of CORM-2 on NF-KB activation in the liver and lung of $E$ coli infected mice

Mice were infected by $E$ coli followed by immediate intravenous administration of CORM-2 or iCORM-2. Six hours after the injection, NF-kB activation in the liver and lung of $E$ coli and $E$ coli+iCORM-2 group mice were significantly increased compared to those of the control group (liver, $0.98 \pm 0.01$ and $0.98 \pm 0.01$ vs $0.53 \pm 0.01$; lung, $1.10 \pm 0.06$ and $1.11 \pm 0.03$ vs $0.54 \pm 0.01, P<0.05)$, while this elevation was significantly inhibited in the $E$ coli+CORM-2 group (liver, $0.77 \pm 0.01$ vs $0.98 \pm 0.01$; lung, $0.87 \pm 0.01$ vs $1.10 \pm 0.06, P<0.05$, Figure 8$)$.

\section{Discussion}

In the United States, the incidence of severe sepsis is estimated to $3 \%$ of the population; septic shock is associated with the highest mortality (approaching 50\%) ${ }^{[21]}$. Sepsis arises from the complex interactions between the infecting microorganism and the host immune, inflammatory and coagulation responses ${ }^{[22]}$. The fundamental pathogenesis of sepsis has not yet been fully elucidated $^{[23,24]}$. Sepsis often progresses in cases where the host cannot contain the primary infection, which is commonly related to the characteristics of the microorganism ${ }^{[25]}$.

Escherichia coli (E coli), which is an important member of the normal intestinal microflora of humans, typically colonizes the mammalian gut within few hours after birth. E coli and its human host coexist in good health and with mutual benefit for decades $^{[26,27]}$. However, during severe sepsis and sepsis shock, human immunity is compromised and the permeability of the intestinal mucosa changes. E coli, as an opportunistic pathogen, can migrate from the gastrointestinal tract to the extraintestinal sites by bacterial translocation ${ }^{[28,29]}$, causing local or disseminated infection and even life-threatening secondary infections.

Many experimental studies have highlighted the specific and independent role of exogenous $\mathrm{CO}$ in modulating inflammation $^{[30,31]}$. As new metal carbonyl-based compounds, CORMs release $\mathrm{CO}$ in biological systems ${ }^{[32]}$. CORM-2, a DMSOsoluble CORM, has been shown to exhibit anti-inflammatory actions and to improve the function of many important organs in CLP and thermal injury models of sepsis in our previous studies $^{[33-35]}$. Therefore, we extended these investigations of the anti-inflammatory effects of exogenous $\mathrm{CO}$ in an $E$ coli (ATCC25922)-induced murine sepsis model.

Previously, we confirmed that intravenous administration of CORM-2 effectively inhibited pulmonary and hepatic pathological injury, MPO activity, expression of TNF- $\alpha$ and IL-1 $\beta$, transcription of iNOS and NF-kB activity in CLP mice. However, whether CORM-2 exerted its effects on directly suppressing the vitality of bacteria remained unknown. To further explore the potential mechanisms, the in vitro growth curve 


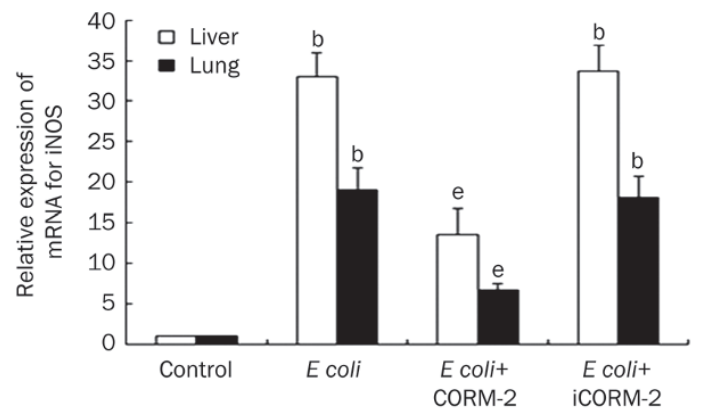

Figure 7. Effect of CORM-2 on iNOS mRNA expression in the liver and lung of $E$ coli infected mice. iNOS mRNA expression was analyzed by RTPCR. At $6 \mathrm{~h}$ after $E$ coli infection, the expression of iNOS mRNA in liver and lung significantly increased compared to that of the control group. This increase was significantly reduced in the $E$ coli+CORM-2 group. $n=8$. Data represent the mean \pm SD. ${ }^{b} P<0.05$ compared with the control group. ${ }^{\mathrm{e}} \mathrm{P}<0.05$ compared with the $E$ coli group.

and colony counting of bacteria were investigated. Growth curve and colony counting were typically used to observe the vitality of bacteria, by which we could monitor the real-time growth of bacteria ${ }^{[36]}$. Results of growth curve and colony counting showed that bacterial growth was significantly suppressed after CORM-2 intervention when compared with the $E$ coli and E coli+iCORM-2 groups, which implied that CORM-2 directly inhibited the bacterial vitality.

Myeloperoxidase (MPO) is an enzyme that is found predominantly in the azurophilic granules of PMNs. The interaction between leukocytes and endothelial cells plays an important role in inflammation and tissue damage ${ }^{[37]}$. Furthermore, the quantitation of tissue MPO activity is a useful biomarker for assessing the influx of $\mathrm{PMNs}^{[38]}$. In the present study, we found that MPO activity in liver and lung tissues was signifi- cantly elevated after bacterial infection and this effect was significantly attenuated after in vivo administration of CORM-2 but not iCORM-2. In accordance with previously reports ${ }^{[15,17]}$, this finding indicated that treatment with CORM-2 effectively prevented PMN chemotaxis and infiltration of liver and lung tissues after bacterial infection, consequently decreasing the production of oxidants, and reducing oxidant-mediated injury and inflammatory response in these tissues.

The data from this study showed that infection with a large number of bacteria induced hepatocellular injury as evidenced by increased plasma ALT and AST levels. Serum ALT and AST levels markedly increased in sepsis mice. However, this effect was not observed in sepsis mice treated with CORM2, indicating CORM-2 attenuates the hepatocellular injury associated with sepsis in this model. Similarly, CORM-2 treatment abolished the elevation in TNF- $\alpha$ and IL-1 $\beta$ levels in the serum and liver and lung tissue homogenates of sepsis mice. TNF- $\alpha$ and IL-1 $\beta$ play a critical roles in the pathogenesis of sepsis and related syndromes. Moreover, infectious diseases can induce exaggerated production of inflammatory cytokines such as TNF- $\alpha$ and IL-1 $\beta$ in the early stages ${ }^{[39]}$. Similar protective effects of CORM-2 on inhibiting TNF- $\alpha$ and IL-1 $\beta$ were discovered in CLP-induced peritonitis sepsis and LPS-induced sterile sepsis ${ }^{[40]}$. Our results suggested that CORM-2 prevents liver damage, and decreases the production of TNF-a and IL-1 $\beta$ in liver and lung following bacterial infection. These data indicate that the cytoprotective effects of CORM-2 are, at least partly, due to inhibition of the production and secretion of TNF- $\alpha$ and IL-1 $\beta$ during the early period of bacterial infection.

The inducible iNOS, which is expressed in macrophages and neutrophils, is a calcium-independent enzyme that is upregulated in response to inflammation ${ }^{[41]}$. Compared with the other isoforms, iNOS activity is more sustained resulting
A

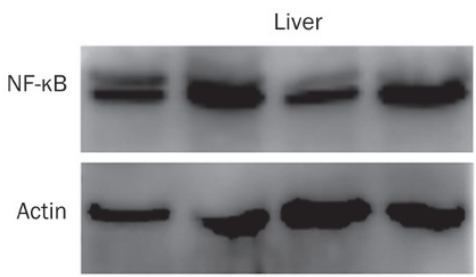

B

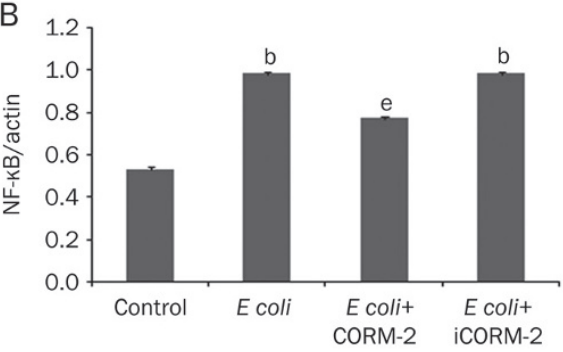

C
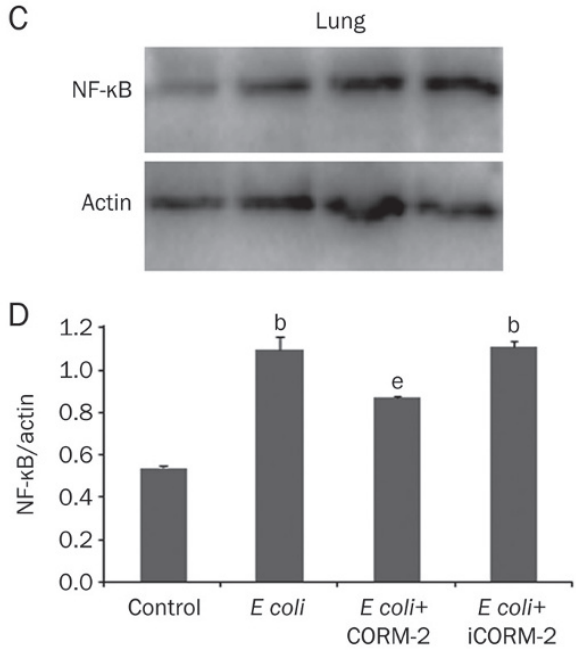

Figure 8. Effects of CORM-2 on NF-KB activation in the liver and lung of $E$ coli infected mice. Mice were infected by $E$ coli followed by immediate intravenous administration of CORM-2 or iCORM-2. Six hours after the injection, NF-KB activation in the liver (A) and lung (B) were significantly increased compared to those of the control group, while this elevation was significantly inhibited in the $E$ coli+CORM-2 group. $n=8$. Data represent the mean \pm SD. ${ }^{\mathrm{b}} P<0.05$ compared with the control group. ${ }^{\mathrm{e}} \mathrm{P}<0.05$ compared with the $E$ coli group. 
in a concomitant increase in nitric oxide $(\mathrm{NO})^{[42]}$. NO plays a major role in cellular injury resulting in the production of reactive oxygen and oxidative stress ${ }^{[43]}$. In this study, we found that bacterial infection produced a significant upregulation in iNOS mRNA expression. After administration of CORM2 , the iNOS mRNA expression was significantly decreased. It can be speculated that this effect leads to inhibition of NO production and amelioration of cellular injury in this model.

The NF-kB family members are ubiquitous, rapidly acting transcription factors that modify immune and inflammatory reactions by regulating the expression of some chemokines and cytokines ${ }^{[44,45]}$. Previously, we showed that CORM-2 plays a pivotal role in the inhibition of NF-kB activity in the liver in thermal injury model in mice, by decreasing the hepatocellular secretion of inflammatory cytokines and hepatic dysfunction $^{[15]}$. Published reports also confirmed that NF-KB activity was significantly down-regulated in LPS-stimulated endothelial cells and macrophages interfered by CORM-2 ${ }^{[46,47]}$. In the present study, NF-KB activity in liver and lung tissues were elevated by bacterial infection, while it was markedly inhibited by administration of CORM-2. All these data showed that CORM-2 plays an even greater role in the inhibition of NF-KB activity, resulting in decreased expression of inflammatory factors such as TNF- $\alpha$, IL- $1 \beta, \mathrm{NO}$, and improved organ function after bacterial infection.

Interestingly, our results of in vivo experiments showed that there were large numbers of $E$ coli in the blood, peritoneal lavage fluid, liver and lung homogenates of mice following bacterial infection. This was accompanied by markedly increased serum LPS concentrations. After administration of CORM-2, both the number of $E$ coli and the concentration of LPS were significantly decreased. The endotoxin LPS, which is a constituent of the cell wall of Gram-negative bacteria, is released on bacterial death. The presence of endotoxins in the blood, known as endotoxemia, can lead to septic shock ${ }^{[48]}$. On the basis of our data, it can be speculated that, rather than causing cell death, CORM-2 inhibits the proliferation of $E$ coli in vivo; however, this remains to be confirmed in future studies.

Here, we observed $100 \%$ mortality in mice within $36 \mathrm{~h}$ of intraperitoneal injection of $E$ coli. CORM-2 administration immediately after bacterial infection significantly increased the survival rate, which reached $55 \%$. This phenomenon was not observed following administration of iCORM-2 in this model and all mice died within $36 \mathrm{~h}$ of bacterial infection. Previously studies and the present data indicated that CORMs might exert a critical therapeutic potential in increasing survival in both sepsis and other severe medical condition such as cardiovascular disease ${ }^{[49,50]}$, and organ transplantation and preservation $^{[51,52]}$.

Taken together these findings indicate that CORM-released CO suppresses Escherichia coli vitality, protects the liver and lungs against $E$ coli-mediated sepsis, resulting in improved survival. However, there is still a great gap in the respect to applying CORM-2 to treatment in clinical sepsis. E coilchallenged murine sepsis model can't fully mimic the patho- physiology in humans. Besides, although $\mathrm{CO}$ gas has already passed safety evaluation in Phase I testing in healthy human beings ${ }^{[53]}$, CORM-2 as a kind of transitional metal carbonyls, metabolical and toxicological effects need to be further characterized.

\section{Acknowledgements}

This study was supported by the National Natural Science Foundation of China (№ 81071546, № 81272148 and № 81471903); and by the Jiangsu Natural Science Foundation, № BK2012703.

\section{Author contribution}

Wei-chang SHEN designed the research and wrote the main manuscript text as the co-first author; Xu WANG designed and analyzed data, and wrote the main manuscript text as the co-first author, Wei-ting QIN performed the research and prepared Figures 1-4; Xue-feng QIU contributed new reagents/ analytic tools and prepared Figures 5-8; Wei-chang SHEN, Xu WANG, and Bing-wei SUN analyzed the data. Bing-wei SUN designed and supervised experiments. He reviewed and submitted the manuscript as the corresponding author. All authors reviewed the manuscript.

\section{References}

1 Dellinger RP, Levy MM, Rhodes A, Annane D, Gerlach H, Opal SM, et al. Surviving sepsis campaign: international guidelines for management of severe sepsis and septic shock: 2012. Crit Care Med 2013; 41: 580-637.

2 Howell G, Tisherman SA. Management of sepsis. Surg Clin N Am 2006; 86: 1523-28.

3 Boomer JS, To K, Chang KC, Takasu O, Osborne DF, Walton AH, et al. Immunosuppression in patients who die of sepsis and multiple organ failure. JAMA 2011; 306: 2594-605.

4 Alberti C, Brun-Buisson C, Burchardi H, Martin C, Goodman S, Artigas $A$, et al. Epidemiology of sepsis and infection in ICU patients from an international multicentre cohort study. Intensive Care Med 2002; 28: 108-21.

5 Angus DC, Linde-Zwirble WT, Lidicker J, Clermont G, Carcillo J, Pinsky MR. Epidemiology of severe sepsis in the United States: analysis of incidence, outcome, and associated costs of care. Crit Care Med 2001; 29: 1303-10.

6 Mayr FB, Yende S, Angus DC. Epidemiology of severe sepsis. Virulence 2014; 5: 4-11.

7 Bukh AS, Schonheyder HC, Emmersen JM, Sogaard M, Bastholm S, Roslev P. Escherichia coli phylogenetic groups are associated with site of infection and level of antibiotic resistance in communityacquired bacteraemia: a 10 year population-based study in Denmark. J Antimicrob Chemother 2009; 64: 163-8.

8 Carlet J, Ben Ali A, Chalfine A. Epidemiology and control of antibiotic resistance in the intensive care unit. Curr Opin Infect Dis 2004; 17: 309-16.

9 Coburn RF. The measurement of endogenous carbon monoxide production. J Appl Physiol 2012; 112: 1949-55.

10 Ryter SW, Choi AM. Carbon monoxide: present and future indications for a medical gas. Korean J Int Med 2013; 28: 123-40.

11 Ozaki KS, Yoshida J, Ueki S, Pettigrew GL, Ghonem N, Sico RM, et al. Carbon monoxide inhibits apoptosis during cold storage and protects kidney grafts donated after cardiac death. Transpl Int 2012; 25: 
107-17.

12 Motterlini R, Mann BE, Johnson TR, Clark JE, Foresti R, Green CJ. Bioactivity and pharmacological actions of carbon monoxide-releasing molecules. Curr Pharm Des 2003; 9: 2525-39.

13 Motterlini R, Clark JE, Foresti R, Sarathchandra P, Mann BE, Green CJ. Carbon monoxide-releasing molecules: characterization of biochemical and vascular activities. Circ Res 2002; 90: E17-24.

14 Liu DM, Sun BW, Sun ZW, Jin Q, Sun Y, Chen X. Suppression of inflammatory cytokine production and oxidative stress by CO-releasing molecules-liberated $\mathrm{CO}$ in the small intestine of thermally-injured mice. Acta Pharmacol Sin 2008; 29: 838-46.

15 Sun BW, Sun Y, Sun ZW, Chen X. Carbon liberated from CO liberated from CORM-2 modulates the inflammatory response in the liver of thermally injured mice. World J Gastroenterol 2008; 14: 547-53.

16 Sun BW, Jin Q, Sun Y, Sun ZW, Chen X, Chen ZY, et al. Carbon liberated from CO-releasing molecules attenuates leukocyte infiltration in the small intestine of thermally injured mice. World J Gastroenterol 2007; 13: 6183-90.

17 Sun B, Sun H, Liu C, Shen J, Chen Z, Chen X. Role of CO-releasing molecules liberated $\mathrm{CO}$ in attenuating leukocytes sequestration and inflammatory responses in the lung of thermally injured mice. J Surg Res 2007; 139: 128-35.

18 Wang X, Qin W, Qiu X, Cao J, Liu D, Sun B. A novel role of exogenous carbon monoxide on protecting cardiac function and improving survival against sepsis via mitochondrial energetic metabolism pathway. Int J Biol Sci 2014; 10: 777-88.

19 Stein AB, Guo Y, Tan W, Wu WJ, Zhu X, Li Q, et al. Administration of a CO-releasing molecule induces late preconditioning against myocardial infarction. J Mol Cell Cardiol 2005; 38: 127-34.

20 Hillegass LM, Griswold DE, Brickson B, Albrightson-Winslow C. Assessment of myeloperoxidase activity in whole rat kidney. J Pharmacol Methods 1990; 24: 285-95.

21 Angus DC, Linde-Zwirble WT, Lidicker J, Clermont G, Carcillo J, Pinsky MR. Epidemiology of severe sepsis in the United States: Analysis of incidence, outcome, and associated costs of care. Crit Care Med 2001; 29: 1303-10.

22 Oberholzer A, Oberholzer C, Moldawer LL. Sepsis syndromes: understanding the role of innate and acquired immunity. Shock 2001; 16 : 83-96.

23 Azevedo LC. The many facets of sepsis pathophysiology and treatment. Shock 2013; 39 Suppl 1: 1-2.

24 Hotchkiss RS, Karl IE. The pathophysiology and treatment of sepsis. New Engl J Med 2003; 348: 138-50.

25 Okazaki Y, Matsukawa A. Pathophysiology of sepsis and recent patents on the diagnosis, treatment and prophylaxis for sepsis. Recent Pat Inflamm Allergy Drug Discov 2009; 3: 26-32.

26 Villa R, Martorana AM, Okuda S, Gourlay LJ, Nardini M, Sperandeo $\mathrm{P}$, et al. The Escherichia coli Lpt transenvelope protein complex for lipopolysaccharide export is assembled via conserved structurally homologous domains. J Bacteriol 2013; 195: 1100-8.

27 Hernandez G, Bruhn A, Ince C. Microcirculation in sepsis: new perspectives. Curr Vasc Pharmacol 2013; 11: 161-9.

28 Hansom D, Littlejohn MG, Clancy MJ. Pyogenic ventriculitis following enteral bacterial translocation in a patient with small bowel obstruction. Scott Med J 2012; 57: 60-5.

29 Citak A, Yilmaz O, Pekcetin C, Ozbal S, Lambrecht FY. Influence of uracil on bacterial translocation in an intestinal obstruction model in rats. Int J Surg 2013; 11: 27-30.

30 Otterbein LE, Bach FH, Alam J, Soares M, Lu HT, Wysk M, et al. Carbon monoxide has anti-inflammatory effects involving the mitogenactivated protein kinase pathway. Nat Med 2000; 6: 422-8.
31 Nakao A, Moore BA, Murase N, Liu F, Zuckerbraun BS, Bach FH, et al. Immunomodulatory effects of inhaled carbon monoxide on rat syngeneic small bowel graft motility. Gut 2003; 52: 1278-85.

32 Motterlini R, Mann BE, Johnson TR, Clark JE, Foresti R, Green CJ. Bioactivity and pharmacological actions of carbon monoxide-releasing molecules. Curr Pharm Des 2003; 9: 2525-39.

33 Sun BW, Sun Z, Jin Q, Chen X. CO-releasing molecules (CORM-2)liberated $\mathrm{CO}$ attenuates leukocytes infiltration in the renal tissue of thermally injured mice. Int J Biol Sci 2008; 4: 176-83.

34 Sun BW, Chen ZY, Chen X, Liu C. Attenuation of leukocytes sequestration by carbon monoxide-releasing molecules: Liberated carbon monoxide in the liver of thermally injured mice. J Burn Care Res 2007; 28: 173-81.

35 Wang X, Cao J, Sun BW, Liu DD, Liang F, Gao L. Exogenous carbon monoxide attenuates inflammatory responses in the small intestine of septic mice. World J Gastroenterol 2012; 18: 5719-28.

36 Bren A, Hart Y, Dekel E, Koster D, Alon U. The last generation of bacterial growth in limiting nutrient. Bmc Syst Biol 2013; 7: 27.

37 Cioffi WG, Burleson DG, Pruitt BA Jr. Leukocyte responses to injury. Arch Surg 1993; 128: 1260-7.

38 Bradley PP, Priebat DA, Christensen RD, Rothstein G. Measurement of cutaneous inflammation: estimation of neutrophil content with an enzyme marker. J Invest Dermatol 1982; 78: 206-9.

39 Kim GY, Roh SI, Park SK, Ahn SC, Oh YH, Lee JD, et al. Alleviation of experimental septic shock in mice by acidic polysaccharide isolated from the medicinal mushroom phellinus linteus. Biol Pharm Bull 2003; 26: 1418-23.

40 Tsoyi K, Lee TY, Lee YS, Kim HJ, Seo HG, Lee JH, et al. Hemeoxygenase-1 induction and carbon monoxide-releasing molecule inhibit lipopolysaccharide (LPS)-induced high-mobility group box 1 release in vitro and improve survival of mice in LPS- and cecal ligation and puncture-induced sepsis model in vivo. Mol Pharmacol 2009; 76: 173-82.

41 Wallace JL, Miller MJ. Nitric oxide in mucosal defense: a little goes a long way. Gastroenterology 2000; 119: 512-20.

42 Saini AS, Shenoy GN, Rath S, Bal V, George A. Inducible nitric oxide synthase is a major intermediate in signaling pathways for the survival of plasma cells. Nat Immunol 2014; 15: 275-82.

43 Stanek A, Gadowska-Cicha A, Gawron K, Wielkoszynski T, Adamek B, Cieslar G, et al. Role of nitric oxide in physiology and pathology of the gastrointestinal tract. Mini-Rev Med Chem 2008; 8: 1549-60.

44 Baeuerle PA, Henkel T. Function and activation of NF-kappa B in the immune system. Ann Rev Immunol 1994; 12: 141-79.

45 Yin MJ, Yamamoto Y, Gaynor RB. The anti-inflammatory agents aspirin and salicylate inhibit the activity of I(kappa)B kinase-beta. Nature 1998; 396: 77-80.

46 Sun B, Zou X, Chen Y, Zhang P, Shi G. Preconditioning of carbon monoxide releasing molecule-derived $\mathrm{CO}$ attenuates LPS-induced activation of HUVEC. Int J Biol Sci 2008; 4: 270-8.

47 Kim BC, Choi JW, Hong HY, Lee SA, Hong S, Park EH, et al. Heme oxygenase-1 mediates the anti-inflammatory effect of mushroom Phellinus linteus in LPS-stimulated RAW264.7 macrophages. J Ethnopharmacol 2006; 106: 364-71.

48 Howard MD, Cox AD, Weiser JN, Schurig GG, Inzana TJ. Antigenic diversity of Haemophilus somnus lipooligosaccharide: phase-variable accessibility of the phosphorylcholine epitope. J Clin Microbiol 2000; 38: 4412-9.

49 Chen B, Guo L, Fan C, Bolisetty S, Joseph R, Wright MM, et al. Carbon monoxide rescues heme oxygenase-1-deficient mice from arterial thrombosis in allogeneic aortic transplantation. Am J Pathol 2009; 175: 422-9. 
50 Chlopicki S, Lomnicka M, Fedorowicz A, Grochal E, Kramkowski K, Mogielnicki $A$, et al. Inhibition of platelet aggregation by carbon monoxide-releasing molecules (CO-RMs): comparison with NO donors. Naunyn Schmiedebergs Arch Pharmacol 2012; 385: 641-50.

51 Sato K, Balla J, Otterbein L, Smith RN, Brouard S, Lin Y, et al. Carbon monoxide generated by heme oxygenase-1 suppresses the rejection of mouse-to-rat cardiac transplants. J Immunol 2001; 166: 4185-94.

52 Nakao A, Choi AMK, Murase N. Protective effect of carbon monoxide in transplantation. J Cell Mol Med 2006; 10: 650-71.

53 Motterlini R, Otterbein LE. The therapeutic potential of carbon monoxide. Nat Rev Drug Discov 2010; 9: 728-43. 